\title{
Unique Establishment of Data Handling and Data Checking in Public Cloud
}

\author{
P. Rajkumar ${ }^{1}$, S. Syedali ${ }^{2}$, K. Vishal ${ }^{3}$, K. Kumaravel ${ }^{4}$ \\ UG, Department of CSE, MKCE, Karur, India ${ }^{1,2,3,4}$
}

\begin{abstract}
In this real world the Cloud Computing, has develop a possible key for sharing Computing resources. Though it has distributing Computing service slightly than having native servers or individual devices to control applications, there are durable protection anxieties in management data. The current system are constructed with rough security ideas but it has some problems. The ideas are similar discrete responsibility, demonstrable data ownership, Third Party Inspecting are secure but it has some issues. To provide enough security in public Cloud a little research has been made on previous idea we suggest a new outline recognized as uniqueness established data handling and data checking in public Cloud which feats the welfares of quantum mechanisms to shelter public database. We took some reference in which rough public Cloud server, owner and client are provided with secure connection with our projected idea which uses arbitrary oracle model. Our logical learning has both achievement and disappointment rates with isolated and common Clouds respectively. Many customers would like to accumulate their information to common Cloud servers laterally with the quick growth of Cloud Computing. New safety issue have to be resolved in order to support more customers process their data in common Cloud.
\end{abstract}

Keywords: Cloud Computing, Management Data, Data handling.

\section{INTRODUCTION}

Cloud Computing is a recently emerged Computing terminology. In this real world the Cloud Computing, has become a feasible solution for sharing Computing possessions. Cloud Computing comprises clusters of isolated servers and software grids that agree data database and internet access to devices. Clouds can be separated as free, isolated or hybrid.

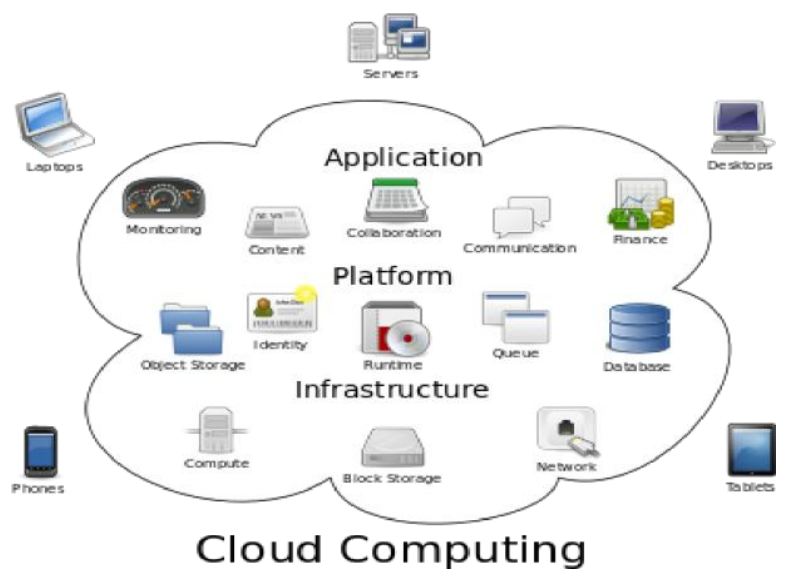

Cloud Computing based on distribution of services to attain logical andfinancial prudenceof scale, like to a efficacy concluded a net. At the base of unrestricted Cloud Computing stays the concept oforganizatied structure anddistributed resources. Cloud Computing, or in simpler just "the Cloud", which too efforts on increasing theusefulness of the distributed services. Cloud allotting different services to multiple handlers. For example, a Cloud computer capability that provides European operators during European working hours with a unique application (e.g., email) which may change the same properties to provide North American operators during North America's working hours with a specific application (e.g., a web server).

This concept should increase the use of systems thus falling ecological damage as it is fewer power, less AC's and space, etc. are required for a different work. In Cloud Computing, many handlers can access one local to recover and update their information without buying authorizations for variety of applications.The term "moving to Cloud" referred to as an company moving away from a outdated model of buy the devoted hardware and depreciate it concluded a certain amount of time to the latest trend of using a common Cloud organizatied structure and pay as one uses it.

Supporters privilege that Cloud Computing permits corporates to avoid unnecessary prices, and focus on missions that distinct their dealings instead of on organizatied structure. They also privilege that Cloud Computing provides organization to get their software running faster, with decent management and fewer maintenance, and permits Information Technology to extra fast adjust capitals to meet rise and fall the unexpected corporate demand. Cloud earners naturally use a "pay as you go" system. It leads to unpredictable high cost if managers do not adjust to the Cloud rating model.

The current accessibility of high-database networks, lowprice pc's and storing devices as well as the wide approval of SOA, and usefulness Computing have leads to a 
development in public Cloud Computing. Cloud database suggestions an on-demand information subcontracting model, and is increasing status due to its springiness and less care and cost. Still, safety problem arises when information database is subcontracted to other Cloud service suppliers. It is necessary to allow Cloud customers to authenticate the veracity of their processed information, in case their information have been tactlessly sullied or any nastily conceded by unknown attacks.

One key issue in use of Cloud database is long-term archival, which gives a load that is carved once and hardly read. While the information is hardly read, it is essential to conform its integrity for adversity retrieval or agreement with allowed requirements. Because it is usual to have a vast quantity of compressed information, full-file testing becomes expensive. Proof of information control have thus been projected to confirm the integrity of a huge file by inspecting only a segment of the file via many cryptographic primitives.

This method lasts to use casual hiding to give information secrecy during open reviewing, and influence guide hash tables to give fully lively actions on shared information. A process indicates an insert, delete or update task on a lone block in public information. CLOUD Computing has been taken as a fresh model of enterprise IT structure, which can form vast service of Computing, database and applications, and enable customers to get global, suitable and grid access to a shared configurable Computing assets with great proficiency and negligible economic price. Attracted by these features, both persons and enterprises are encouraged to upload their information to the Cloud, instead of buying software and hardware to succeed the information themselves.

In spite of of the several rewards of Cloud services, subcontracting subtle data such as electronic mail, individual health archives, company investment data, administration forms, etc. to isolated servers carries some confidentiality anxieties. The Cloud amenity workers keep the info for handlers may admission delicate information without any approval. A all-purpose idea to defend the information privacy is to encrypt the information previously uploading. However, this determination reason a huge price in footings of statistics usability.

\section{LITERATURE SURVEY}

\section{OVERVIEW}

A work study is an explanation of pardon has been available on a matter by credited intellectuals and investigators. Infrequently you resolve be enquired to inscribe one as a isolated obligation, but extra regularly it is slice of the outline to a interpretation, enquiry or thesis. In writing the works appraisal, your resolve is to carry to your bibliophile what data and ideas have been reputable on a description, and what their optimistic and undesirable area. As per a part of script, the work appraisal must be well-defined by a managerial idea (e.g., your study impartial, the matter you interchange or inscribe your belligerent thesis)

Also expanding your information round the area, inscription a work review occupancies you improvement and reveal services in two parts

1. INFORMATION SEEKING: the skill to image the works proficiently, using physical or electronic methods, to find a set of valuable tutelages and files

2. CRITICAL APPRAISAL: the skill to rub on philosophies of analysis to find impartial and legal studies.

\section{ACHIEVING EFFICIENT CLOUD SEARCH} SERVICES MULTIEYWORD RANKED SEARCH OVER ENCRYPTED CLOUD DATA SUPPORTING PARALLEL COMPUTING

In few eons, consumer-centric Cloud Computing architype innovative as per the progress of keen electronic plans mutual by the new Cloud Computing tools. A variability of Cloud services stay hand over to the users per the foundation that actual Cloud examination package is achieved. In lieu of consumers, they need to discover the most applicable produces or data, which is extremely useful in the "pay-as-you use" Cloud Computing. Equal complex information is encrypted earlier outside supplier near Cloud, outdated keyword rifle techniques are useless. Meanwhile, existing search standard or above encoded Cloud data provision only strict before unsure keyword pursuit, then not semantics-based multi tiered looking. Therefore, in what way to authorize to produced desired searchable scheme with care of ranked rifle relics a actual defend tricky. This rag suggests a wanted attitude to explain the problematic of multikeyword sorting search from encoded Cloud information stand all replacement inquiries. The main involvement of this paper is potted in two features: multi-keyword tiered quest to accomplish more exact seeking effects and phrase-based pursuit to funding substitute enquiries. General tries on actual dataset were achieved to check the tactic, viewing that the projected key is actual expense aimed at multikey word graded probing in a Cloud atmosphere.

\section{A PROXY RE-ENCRYPTION SYSTEM WITH THE UNFORGEABILITY OF RE-ENCRYPTION KEYS AGAINST COLLUSION ATTACKS}

Proxy re-encryption (PRE) schemes are cryptosystems which allow a proxy who has a re-encryption important to convert a cipher script originally encrypted meant for one party addicted to a cryptogram text which can be decrypted by another party. In Hayashi et al. proposed the new security desire for PRE called "unforgeability of reencryption keys against secret attacks ," UFReKey-CA for short. They proposed the PRE plan and claimed that their systematic plan acquaintance UFReKey-CA. However, Isshikietal. pointed out that the schemes do not meet UFReKey-Ca in IWSEC2013. It is an open problem of erect the plan which meets UFReKey-CA. In this paper, we propose new PRE plan which meet confidentiality 
(RCCA security) assuming that the q-wDBDHI problem is hard and meet UFReKey-CA assuming that the 2-DHI problem is hard.

\section{PROXY RE-ENCRYPTION FROM LATTICES}

We legislature a original unidirectional proxy reencryption secret founded on the rigidity of the LWE problem. Our structure is agreement innocuous and does not need any important specialist for the re-encryption key group. We outspread a new trapdoor meaning for a square of McConnico and Peaker. Our proxy re-encryption arrangement stays provably CCA-1 safe in the careful model below the LWE supposition.

\section{MUTUAL VERIFIABLE PROVABLE AUDITING IN PUBLIC CLOUD DATABASE}

Cloud database is here and now a popular investigation issue in information technology. In Cloud database, data protection things such as information privacy, truth and convenience become additional and extra significant in many future applications. Recently, several trustable information possession (PDP) structures are projected to guard information honour. In some gears, It has to approved the isolated information switch scrutiny task to some representation.

However, these PDP systems are not safe since the proxy fatty some state-run information in Cloud database servers. effect of this paper is summarized in two aspects: multi-keyword ranked pursuit to Hence, trendy this paper, we suggest an outlay skilled provable information control plan, which uses Diffie-Hellman a share key to knowledge the procedural authenticator. In specific, the true popular our plan is displaced and autonomous of the Cloud database service. It stays item noticing that the offered system is actual effective rate by the earlier PDP plan, subsequently the bilinear process is not mandatory.

\section{CURRENT SYSTEM}

In Cloud Computing, the customers store their huge information in the isolated public Cloud servers. In the interim the deposited information is external of the controller of the customers, it requires the protected menaces in terms of privacy, veracity and convenience of information and service. In this manager Cloud not process entire client, hence the administrator has to representative the proxy to procedure its information. Public scrutiny will suffer some risk of leaky the secrecy. To overcome the privacy, issue remote data integrity checking protocol is introduced to perform the certificate management. Acceptable expenditure derives from the heavy documents substantiation, credentials cohort, distribution, reversal, restitutions, etc.

\section{SHORTCOMINGS OF THE CURRENT SYSTEM:}

- The security is in threats, in standing of privacy, truthfulness and handiness of information while subcontract the data toward Cloud database.
Certificate management cause heavy computational overheads

- User privacy is not achieved

\section{PROPOSED SYSTEM}

Cloud Computing, an innovative prototypical of Computing, has develop a truth which allows information holders to subcontract their information to public Cloud too providing various additional services. However, the Cloud attendants are dried "untrusted" by Cloud customer as their valued information is stored in isolated servers. There are several security fears over the subcontracted information and structures between the Cloud server and Cloud handlers.

Many solutions came into presence in demand to limit this badly-behaved. Future a brink proxy re-encryption system that fortifies subcontracted statistics. Their security planning is simplified by number of database attendants and key servers. The database servers store information while the key servers turn as admission nodes. The system cares encoding, encryption and progressing. Each database server and key server autonomously makes encoding and re-encryption and part decryption individually. In common Cloud, this structure efforts on the uniqueness established data handling and data checking in public cloud. It is based secure-based public key cryptology, is effective since the credential managing is removed.

\section{ADVANTAGE OF PROPOSED SYSTEM}

- The computation and communication overhead are achieved

- Our proposed protocol mollifies the isolated read through, proxy checking and common checking

- CDH problem is solved

- Outsourced data security is achieved

- Time efficiency is achieve

\section{CONCLUSION}

In this project, we planned a outline for protected Cloud Computing. The outline is based on individual major and standard cryptography. It simplifies both major channel and information channel for shifting key and information respectively. The safety framework built on important cryptography planned in our last paper is reprocessed to some level to construct the new framework. Safety encryption is a method that allows secure examine over encrypted information stored on distant servers. In our future slog, we created a new secure and competent multikeyword likeness searchable encryption that yields the similar information things from the Cloud server.

\section{REFERENCES}

[1] S Saravanan, V Venkatachalam, S Then Malligai "Optimization of SLA violation in cloud computing using artificial bee colony" 2015 , 1(3), 323 -327 ISSN: 2394-9260, pp:410-414. 
[2] Z. Fu, X. Sun, Q. Liu, L. Zhou, J. Shu, "Achieving efficient cloud searchservices: multi-keyword ranked search over encrypted Cloud data supporting parallel Computing," IEICE Transactions on Communications, vol. E98-B, no. 1, pp.190-200, 2015.

[3] Y. Ren, J. Shen, J. Wang, J. Han, S. Lee, "Mutual verifiable provable data auditing in public Cloud database," Journal of Internet Technology, vol. 16, no. 2, pp. 317-323, 2015

[4] E. Kirshanova, "Proxy re-encryption from lattices", PKC 2014, LNCS 8383, pp. 77-94, 2014.

[5] H. Guo, Z. Zhang, J. Zhang, "Proxy re-encryption with unforgeable reencryption keys", Cryptology and Network Security, LNCS 8813, pp. 20-33, 2014. [5] P. Xu, H. Chen, D. Zou, H. Jin, "Fine-grained and heterogeneous proxy re-encryption for secure Cloud database", Chinese Science Bulletin, vol.59,no.32, pp. 4201-4209, 2014.

[6] E. Esiner, A. K"upc, "u, "O "Ozkasap, "Analysis and optimization on FlexDPDP: a practical solution for dynamic provable data possession", Intelligent Cloud Computing, LNCS 8993, pp. 65-83, 2014.

[7] H. Wang, "Identity-based distributed provable data possession in multiCloud database", IEEE Transactions on Services Computing, vol. 8, no. 2, pp. 328-340, 2015

[8] H. Wang, "Anonymous multi-receiver remote data retrieval for pay-tv in public Clouds", IET Information Security, vol. 9, no. 2, pp. 108-118, 2015.

[9] Q. Zheng, S. Xu, "Fair and dynamic proofs of retrievability", CODASPY' 11, pp. 237-248, 2011. 